\title{
Effect of the magnetic field on the photon radiation from quark-gluon plasma in heavy ion collisions
}

\author{
B. G. Zakharov ${ }^{\mathrm{a}}$ \\ L.D. Landau Institute for Theoretical Physics, GSP-1, 117940, Kosygina Str. 2, 117334 Moscow, Russia
}

Received: 18 July 2016 / Accepted: 19 October 2016 / Published online: 7 November 2016

(c) The Author(s) 2016. This article is published with open access at Springerlink.com

\begin{abstract}
We develop a formalism for the photon emission from the quark-gluon plasma with an external electromagnetic field. We then use it to investigate the effect of the magnetic field on the photon emission from the quark-gluon plasma created in $A A$ collisions. We find that even for a very optimistic assumption on the magnitude of the magnetic field generated in $A A$ collisions its effect on the photon emission rate is practically negligible. For this reason the magnetic field cannot generate a significant azimuthal asymmetry in the photon spectrum.
\end{abstract}

\section{Introduction}

There is now a variety of experimental data on hadronic observables in $A A$ collisions at RHIC and LHC that show that hadron production in high energy $A A$ collisions goes via formation of a hot quark-gluon plasma (QGP) fireball. The major arguments in favor of the QGP formation at RHIC and LHC are the observation of a strong suppression of high- $p_{T}$ particle spectra (the so-called jet quenching phenomenon) and the success of the hydrodynamical models in describing the flow effects in hadron production in $A A$ collisions. The results of the jet quenching [1-7] and hydrodynamical [8] analyses support the production time of the QGP $\tau_{0} \sim 0.5-1$ $\mathrm{fm}$. However, this is only a qualitative estimate, because the value of $\tau_{0}$ is not well constrained by the data on the jet quenching and the flow effects. For jet quenching it is due to a strong reduction of the radiative parton energy loss in the initial stage of the QGP evolution by the finite size effects $[9,10]$. For this reason jet quenching is not very sensitive to the first $\mathrm{fm} / \mathrm{c}$ of the matter evolution. For the flow effects it is due to the low transverse velocities in the initial stage of the fireball evolution and the correlations of $\tau_{0}$ with the viscosity of the QGP in the hydrodynamical fits $[11,12]$.

\footnotetext{
a e-mail: bgz@itp.ac.ru
}

It is believed that the photon spectrum in the low and intermediate $k_{T}$ region may be more sensitive to the initial stage of the QGP evolution than the hadronic observables. Because the thermal photons radiated from the QGP leave the fireball without attenuation and the photon emission rate is largest in the initial hottest stage of the QGP evolution [13]. The measurements of the photon spectrum in $A A$ collisions performed at RHIC [14-16] and LHC [17] show that there is some excess of the photon yield (above the photons from hadron decays and from the hard perturbative mechanism) at $k_{T} \lesssim 3-4 \mathrm{GeV}$. It is widely believed that it is related to the photon emission from the QGP. However, the results of pQCD calculations of the thermal contribution to the photon spectrum are only in a qualitative agreement with the data obtained at RHIC and LHC (see [18] and references therein). Say, the theoretical predictions obtained in recent analysis [19] using a sophisticated viscous hydrodynamical model of the fireball evolution underestimate the photon spectrum by a factor of $\sim 1.5-3.5$. It was observed that the thermal photons exhibit a significant azimuthal asymmetry $v_{2}$ (elliptic flow) comparable to that for hadrons. It is difficult to reconcile this fact with the expectation that the thermal photons should be mostly radiated from the hottest initial stage of the QGP where the flow effects should be small (this is often called the direct photon puzzle). It was suggested [20] that in the standard pQCD scenario of the thermal photon emission the flow effect for photons may be related to the viscous effects in the QGP that lead to a deviation of the parton distribution functions in the QGP from the equilibrium ones. The numerical results of [19] show that the viscosity of the QGP may be an important source of the photon momentum anisotropy. However, in the analysis [19] the viscous effects have been accounted for only for the LO pQCD $2 \rightarrow 2$ processes $q(\bar{q}) g \rightarrow \gamma q(\bar{q})$ (Compton) and $q \bar{q} \rightarrow \gamma g$ (annihilation), and have not been included for the higher order collinear processes $q \rightarrow \gamma q$ and $q \bar{q} \rightarrow \gamma$ [21]. 
The direct photon puzzle stimulated searches for novel mechanisms of the photon production in $A A$ collisions that could generate a significant azimuthal asymmetry. In Ref. [22] it was suggested that the large photon azimuthal anisotropy may be related to a novel photon production mechanism stemming from the conformal anomaly and a strong magnetic field in noncentral $A A$ collisions. However, the contribution of this mechanism becomes important only for a sufficiently large magnitude of the magnetic field, which is not supported by calculations for realistic evolution of the plasma fireball [23]. In Ref. [24] it was argued that the observed photon asymmetry may be due to an intensive bremsstrahlung like synchrotron radiation resulting from the interaction of escaping quarks with the collective confining color field at the surface of the QGP. For this mechanism the asymmetry arises due to bigger surface emission from the almond-shaped QGP fireball along the direction of the impact parameter vector (as shown in Fig. 1). In Ref. [25] it was suggested that the significant photon $v_{2}$ can be related to the real synchrotron emission from the thermal quarks in a strong magnetic field generated in noncentral $A A$ collisions. Since the magnetic field in the noncentral $A A$ collisions is mostly perpendicular to the reaction plane (this direction corresponds to $y$ axis, if $x$ axis is directed along the impact parameter of the $A A$ collision as shown in Fig. 1) the synchrotron radiation rate is largest in the direction along of the impact parameter vector.

For this reason the synchrotron mechanism leads naturally to a strong azimuthal asymmetry of the photon emission. This explanation works only if the contribution of the synchrotron mechanism to the photon emission rate is significant. The analysis of Ref. [25] shows that in the central rapidity region at $k_{T} \sim 1-3 \mathrm{GeV}$ the contribution of the synchrotron mechanism may be comparable with the observed photon yield in $\mathrm{Au}+\mathrm{Au}$ collisions at $\sqrt{s}=0.2 \mathrm{TeV}$. However, the calculations of Ref. [25] are of a qualitative nature. In [25] the calculations are performed for purely synchrotron radiation. But in the QGP each quark undergoes multiple scattering due to interaction with other thermal quarks and gluons. One can expect that it will lead to a reduction of the coherence/formation length of the photon emission, and

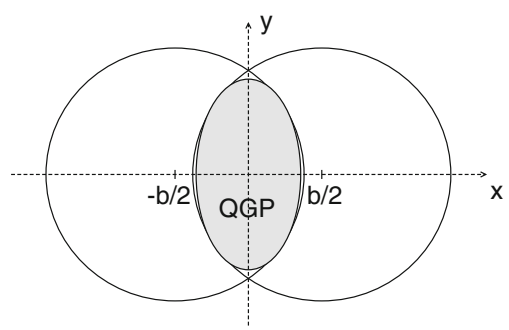

Fig. 1 The transverse plane of a noncentral $A A$-collision with the impact parameter $b$ to suppression of the synchrotron emission. In reality for the QGP with magnetic field one simply cannot distinguish between the synchrotron radiation and the bremsstrahlung due to multiple scattering, and one has to treat the two mechanisms on an even footing. In this case the effect of the magnetic field on the photon emission can only be defined as the difference between the photon emission rate from the QGP with and without magnetic field. Also, in [25] the comparison with the experimental photon spectrum has been performed by integrating over the QGP four volume neglecting the longitudinal and transverse expansion of the QGP. The neglect of the longitudinal expansion of the QGP may be too crude an approximation. For a QGP with zero velocity the energy of a quark radiating a photon with a given momentum is smaller than that in the comoving frame for the QGP with the longitudinal expansion. Since the quark (antiquark) thermal distribution decreases exponentially with quark energy, the approximation of zero QGP velocity can overestimate considerably the photon spectrum. Another issue that can result in overestimation of the synchrotron contribution is the use in [25] of the current quark masses. In the QGP quarks acquire a thermal quasiparticle mass $\sim g T$, which appears after the Hard Thermal Loop (HTL) resummation (which is very important already for the LO $2 \rightarrow 2$ processes [26]). Since the synchrotron spectrum reduces with the charged particle mass, the accounting of the quark quasiparticle mass, which is much bigger than the current quark masses, should considerably suppress the effect of the magnetic field.

Besides the photon bremsstrahlung addressed in [25] the magnetic field can affect the photon production via the annihilation mechanism $q \bar{q} \rightarrow \gamma$. The analysis of the collinear processes $q \rightarrow \gamma q$ and $q \bar{q} \rightarrow \gamma$ for the QGP without magnetic field shows that the annihilation contribution is even more important than bremsstrahlung at the photon momenta $k \gg T$ [21]. The purpose of the present work is to address the effect of the magnetic field on the two processes $q \rightarrow \gamma q$ and $q \bar{q} \rightarrow \gamma$ (below we will call the magnetic field modification for both these processes the synchrotron contribution). We develop a formalism which treats on an even footing the effect of multiple scattering and curvature of the quark trajectories in the collective magnetic field in the QGP. Our analysis is based on the light cone path integral (LCPI) formalism [2730], which was previously successfully used [31] for a very simple derivation of the photon emission rate from the higher order collinear processes $q \rightarrow \gamma q$ and $q \bar{q} \rightarrow \gamma$ obtained earlier by Arnold, Moore and Yaffe (AMY) [21] using methods from thermal field theory with the HTL resummation. It is known that the higher order diagrams corresponding to these processes contribute to leading order [32], and turn out to be as important as the LO $2 \rightarrow 2$ processes $q(\bar{q}) g \rightarrow \gamma q(\bar{q})$ and $q \bar{q} \rightarrow \gamma g$. Contrary to the collinear processes the LO processes should not be affected by the presence of an external magnetic field. Our results differ drastically from that of 
[25]. We find that even for very optimistic magnitude of the magnetic field for RHIC and LHC conditions its effect on the photon emission from the QGP is very small.

The plan of the paper is as follows. In Sect. 2 we first discuss the physical picture of the processes $q \rightarrow \gamma q$ and $q \bar{q} \rightarrow \gamma$. We show that for the magnitude of the magnetic field of interest for $A A$ collisions these process remain in the collinear regime. Then we develop a formalism for evaluation of their contribution to the photon emission from the QGP with magnetic field in the medium rest frame. In Sect. 3 we discuss how to compute the photon spectrum from the plasma fireball in $A A$ collisions. We discuss the model of the fireball and the possible magnitude of the magnetic field for the most optimistic scenario for the synchrotron photon emission. In Sect. 4 we present our numerical results. Section 5 summarizes our work. Some of our results concerning the photon emission rate from the QGP at rest have been reported in an earlier short communication [33].

\section{Bremsstrahlung and pair annihilation in the QGP with magnetic field}

In this section we discuss the photon emission rate per unit time and volume in the equilibrium QGP with magnetic field in the QGP rest frame. Similarly to the analyses [21,31] of the processes $q \rightarrow \gamma q$ and $q \bar{q} \rightarrow \gamma$ for zero magnetic field we treat quarks and photons as relativistic quasiparticles with energies much larger than their quasiparticle masses $m_{q}$ and $m_{\gamma} \cdot{ }^{1}$ For the weakly coupled QGP with $N_{f}$ flavors $m_{q}$ and $m_{\gamma}$ read [21]

$m_{q}=g T / \sqrt{3}$,

$m_{\gamma}=\frac{e T}{3} \sqrt{\left(3+N_{f}\right) / 2}$

where $g=\sqrt{4 \pi \alpha_{s}}$ is the QCD coupling constant, $e$ is the electron charge. In numerical calculations we take $N_{f}=2.5$ to account for qualitatively the suppression of strange quarks at moderate temperatures. Since $m_{q} / m_{\gamma}>>1$ the effect of the nonzero photon mass is very small, and our results are close to that for massless photon.

\subsection{Physical picture of photon emission and photon formation length}

The physical picture behind the derivation of the photon emission rate in the QGP without magnetic field from the processes $q \rightarrow \gamma q$ and $q \bar{q} \rightarrow \gamma$ given in $[21,31]$ is the fact

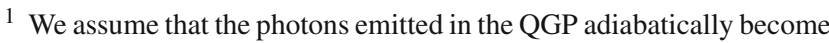
massless after escaping from the plasma fireball.
}

that in the weakly coupled QGP the hard partons with energy $E \gtrsim T$ undergo typically only small angle multiple scattering due to interaction with the random soft gluon fields at the momentum scale $\sim g T$. And the large angle scattering with the momentum transfer $\sim E$ is a very rare process. The typical quark scattering angle at the longitudinal scale about the photon coherence/formation length, $L_{f}$, is small [31]. Due to this fact the processes $q \rightarrow \gamma q$ and $q \bar{q} \rightarrow \gamma$ are dominated by the collinear configurations, when the photon is emitted practically in the direction of the initial quark for $q \rightarrow \gamma q$ (and in the direction of the momentum of the $q \bar{q}$ pair for $q \bar{q} \rightarrow \gamma)$. For a QGP with magnetic field this picture will remain valid if

$\frac{L_{f}}{R_{L}} \ll 1$

where $R_{L}=E_{q} / z_{q} e B$ is the quark Larmor radius in the magnetic field $\left(z_{q}\right.$ is the quark electric charge in units of $e$ ). Let us demonstrate that the condition (3) is satisfied for the fields $e B=c m_{\pi}^{2}$ with $c \lesssim 1$, which are of interest for $A A$ collisions. Making use the formulas of the LCPI approach for the bremsstrahlung due to multiple scattering [27-30] and for the synchrotron emission [34] one can obtain qualitative estimate

$L_{f} \sim \min \left(L_{1}, L_{2}\right)$,

where the quantities $L_{1,2}$ read

$L_{1} \sim \frac{2 E_{q}(1-x) S_{\mathrm{LPM}}}{m_{q}^{2} x}$,

$L_{2} \sim\left(\frac{24 E_{q} x(1-x)}{f^{2}}\right)^{1 / 3}$.

Here $S_{\text {LPM }}$ is the suppression factor due to the LandauPomeranchuk-Migdal (LPM) effect [35,36], $x$ is the photon fractional longitudinal momentum, $f=z_{q} x e B$. For $S_{\mathrm{LPM}}=1$ (4) gives simply the formation length for the synchrotron emission in vacuum [34]. The LPM suppression factor can easily be estimated in the oscillator approximation corresponding to the description of multiple scattering in terms of the transport coefficient $\hat{q}$ in the BDMPS [3840] approach to the induced gluon emission. In the oscillator approximation $S_{\mathrm{LPM}} \sim \frac{3}{\kappa \sqrt{2}}($ see below $(51))$ [27-30], where $\kappa=\left[8 \hat{q} E_{q}(1-x) / 9 x m_{q}^{4}\right]^{1 / 2}\left(\right.$ we take here $\left.m_{\gamma}=0\right)$ A qualitative pQCD estimate gives $\hat{q} \sim 14 T^{3}$ (see below). From the point of view of the photon emission from the QGP the interesting $x$-region is $x \gtrsim 0.5$. Making use of (5), (6) one can obtain for $e B=c m_{\pi}^{2}$ at $x \sim 0.5$ for $u$ quark $\left(z_{q}=2 / 3\right)$

$L_{1} \sim \frac{1}{T} \sqrt{E_{q} / T}$ 
$L_{2} \sim 4\left(\frac{E_{q}}{c^{2} m_{\pi}^{4}}\right)^{1 / 3}$.

From (7) and (8) one can see that for the QGP temperatures $T \gtrsim T_{c}$ (here $T_{c} \approx 160-170 \mathrm{MeV}$ is the deconfinement temperature [37]) we have $L_{1}<L_{2}$ in the energy region of interest $E_{q} \lesssim 5 \mathrm{GeV}$, i.e. we have $L_{f} \sim L_{1}$. Then we obtain

$\frac{L_{f}}{R_{L}} \sim z_{q} c\left(\frac{m_{\pi}}{T}\right)^{3 / 2}\left(\frac{m_{\pi}}{E_{q}}\right)^{1 / 2}$

From (9) for $c=1$ we obtain at $E_{q} \gtrsim 1 \mathrm{GeV}$ for the $u$ quark $L_{f} / R_{L} \lesssim 0.25\left(m_{\pi} / T\right)^{3 / 2}$. Thus the condition (3) is reasonably satisfied even at $T \sim T_{c}$. The contribution of the annihilation $q \bar{q} \rightarrow \gamma$ may be expressed via the spectrum of the $\gamma \rightarrow q \bar{q}$ transition (see below). By repeating the above estimates for $\gamma \rightarrow q \bar{q}$ one can show that for this case the condition (3) is also satisfied.

\subsection{Basic formulas}

The above analysis shows that, similarly to the QGP without magnetic field [21,31], for the QGP produced in $A A$ collisions in the presence of the magnetic field we can treat the processes $q \rightarrow \gamma q$ and $q \bar{q} \rightarrow \gamma$ as the collinear ones. And the contribution of these processes to the photon emission rate per unit time and volume in the plasma rest frame can be written as $[21,31]$

$\frac{\mathrm{d} N}{\mathrm{~d} t \mathrm{~d} V \mathrm{~d} \mathbf{k}}=\frac{\mathrm{d} N_{b r}}{\mathrm{~d} t \mathrm{~d} V \mathrm{~d} \mathbf{k}}+\frac{\mathrm{d} N_{a n}}{\mathrm{~d} t \mathrm{~d} V \mathrm{~d} \mathbf{k}}$

where the first term corresponds to $q \rightarrow \gamma q$ and the second one to $q \bar{q} \rightarrow \gamma$. The bremsstrahlung contribution reads [31]

$$
\begin{aligned}
& \frac{\mathrm{d} N_{b r}}{\mathrm{~d} t \mathrm{~d} V \mathrm{~d} \mathbf{k}}=\frac{\mathrm{d}_{b r}}{k^{2}(2 \pi)^{3}} \sum_{s} \\
& \quad \times \int_{0}^{\infty} \mathrm{d} p p^{2} n_{F}(p)\left[1-n_{F}(p-k)\right] \theta(p-k) \frac{\mathrm{d} P_{q \rightarrow \gamma q}^{s}(\mathbf{p}, \mathbf{k})}{\mathrm{d} k \mathrm{~d} L},
\end{aligned}
$$

where $\mathrm{d}_{b r}=4 N_{c}$ is the number of the quark and antiquark states,

$n_{F}(p)=\frac{1}{\exp (p / T)+1}$

is the thermal Fermi distribution, and $\mathrm{d} P_{q \rightarrow \gamma q}^{s}(\mathbf{p}, \mathbf{k}) / \mathrm{d} k \mathrm{~d} L$ is the probability distribution of the photon emission in the QGP per unit length from a fast quark of type $s$. Since we work in the small angle approximation, we can take the vectors $\mathbf{p}$ and $\mathbf{k}$ parallel. The quantity $d P_{q \rightarrow \gamma q}^{s}(\mathbf{p}, \mathbf{k}) / \mathrm{d} k \mathrm{~d} L$ should be evaluated accounting for the quark interaction with the random soft gluon field generated by the thermal partons and with the smooth external electromagnetic field.

The annihilation contribution can be expressed via the probability distribution for the photon absorption $d N_{a b s} / \mathrm{d} t$ $\mathrm{d} V \mathrm{~d} \mathbf{k}$ with the help of the detailed balance principle, which gives [31]

$\frac{\mathrm{d} N_{a n}}{\mathrm{~d} t \mathrm{~d} V \mathrm{~d} \mathbf{k}}=\left[1+n_{B}(k)\right]^{-1} \frac{\mathrm{d} N_{a b s}}{\mathrm{~d} t \mathrm{~d} V \mathrm{~d} \mathbf{k}}$,

where $n_{B}(k)=1 /[\exp (k / T)-1]$ is the Bose distribution. The photon absorption rate on the right-hand side of (13) can be written as

$$
\begin{aligned}
& \frac{\mathrm{d} N_{a b s}}{\mathrm{~d} t \mathrm{~d} V \mathrm{~d} \mathbf{k}}=\frac{\mathrm{d}_{a n} n_{B}(k)}{(2 \pi)^{3}} \sum_{s} \\
& \quad \times \int_{0}^{\infty} \mathrm{d} p\left[1-n_{F}(p)\right]\left[1-n_{F}(k-p)\right] \theta(k-p) \frac{\mathrm{d} P_{\gamma \rightarrow q \bar{q}}^{s}(\mathbf{k}, \mathbf{p})}{\mathrm{d} p \mathrm{~d} L},
\end{aligned}
$$

where $\mathrm{d}_{a n}=2$ is the number of the photon helicities, $\mathrm{d} P_{\gamma \rightarrow q \bar{q}}^{s}(\mathbf{k}, \mathbf{p}) / \mathrm{d} p \mathrm{~d} L$ is the probability distribution per unit length for the $\gamma \rightarrow q \bar{q}$ transition ( $p$ is the quark momentum and $k-p$ is the antiquark momentum, and similarly to $q \rightarrow \gamma q$ we can take the vectors $\mathbf{p}$ and $\mathbf{k}$ parallel). Using the relation

$$
\begin{aligned}
& \frac{n_{B}(k)}{1+n_{B}(k)}\left[1-n_{F}(p)\right]\left[1-n_{F}(k-p)\right] \\
& \quad=n_{F}(p) n_{F}(k-p)
\end{aligned}
$$

from (13), (14) one obtains [31]

$$
\begin{aligned}
& \frac{\mathrm{d} N_{a n}}{\mathrm{~d} t \mathrm{~d} V \mathrm{~d} \mathbf{k}}=\frac{\mathrm{d}_{a n}}{(2 \pi)^{3}} \sum_{s} \\
& \quad \times \int_{0}^{\infty} \mathrm{d} p n_{F}(p) n_{F}(k-p) \theta(k-p) \frac{\mathrm{d} P_{\gamma \rightarrow q \bar{q}}^{s}(\mathbf{k}, \mathbf{p})}{\mathrm{d} p \mathrm{~d} L} .
\end{aligned}
$$

Let us consider first calculation of the bremsstrahlung contribution. In the LCPI formalism [27-30] the probability of the $q \rightarrow \gamma q$ transition (for a quark with charge $z_{q} e$ ) per unit length can be written in the form (we use here the fractional photon momentum $x$ instead of $k$ )

$$
\begin{aligned}
& \frac{\mathrm{d} P_{q \rightarrow \gamma q}}{\mathrm{~d} x d L}=2 \operatorname{Re} \int_{0}^{\infty} \mathrm{d} z \exp \left(-i \frac{z}{\lambda_{f}}\right) \hat{g}(x)\left[\mathcal{K}\left(\rho_{2}, z \mid \rho_{1}, 0\right)\right. \\
& \left.-\mathcal{K}_{\text {vac }}\left(\boldsymbol{\rho}_{2}, z \mid \boldsymbol{\rho}_{1}, 0\right)\right]\left.\right|_{\rho_{1,2}=0},
\end{aligned}
$$

where $\lambda_{f}=2 M(x) / \epsilon^{2}$ with $M(x)=E_{q} x(1-x), \epsilon^{2}=$ $m_{q}^{2} x^{2}+m_{\gamma}^{2}(1-x)$ (in general for $a \rightarrow b+c$ transition $\left.\epsilon^{2}=m_{b}^{2} x_{c}+m_{c}^{2} x_{b}-m_{a}^{2} x_{b} x_{c}\right), \hat{g}$ is the vertex operator, 
given by

$\hat{g}(x)=\frac{V(x)}{M^{2}(x)} \frac{\partial}{\partial \rho_{1}} \cdot \frac{\partial}{\partial \rho_{2}}$

with

$V(x)=z_{q}^{2} \alpha_{e m}\left(1-x+x^{2} / 2\right) / x$,

$\alpha_{e m}=e^{2} / 4 \pi$ the fine-structure constant. $\mathcal{K}$ in (17) is the retarded Green function of a two dimensional Schrödinger equation, in which the longitudinal coordinate $z$ (along the initial quark momentum) plays the role of time, with the Hamiltonian

$\hat{\mathcal{H}}=-\frac{1}{2 M(x)}\left(\frac{\partial}{\partial \rho}\right)^{2}+v(\rho)$,

and

$\mathcal{K}_{v a c}\left(\rho_{2}, z \mid \rho_{1}, 0\right)=\frac{M(x)}{2 \pi i z} \exp \left[\frac{i M(x)\left(\rho_{2}-\rho_{1}\right)^{2}}{2 z}\right]$

is the Green function for $v=0$. The potential $v$ can be written as

$v=v_{f}+v_{m}$,

where $v_{f}$ is due to the fluctuating gluon fields of the QGP, and $v_{m}$ is related to the mean electromagnetic field. The mean field component of the potential reads

$v_{m}=-\mathbf{f} \rho$,

where $\mathbf{f}=x z_{q} \mathbf{F}, \mathbf{F}$ is transverse component (to the parton momentum) of the Lorentz force for a particle with charge $e$. The effect of the longitudinal Lorentz force (which exists for nonzero electric field) is small for the relativistic partons, and we neglect it. The component $v_{f}$ reads

$v_{f}=-i P(x \rho)$.

Here the function $P(\rho)$ can be written as

$P(\rho)=g^{2} C_{F} \int_{-\infty}^{\infty} \mathrm{d} z\left[G\left(z, 0_{\perp} z\right)-G(z, \rho, z)\right]$,

where $g$ is the QCD coupling, $C_{F}=4 / 3$ is the quark Casimir operator, $G$ is the gluon correlator (the color indices are omitted)

$G(x-y)=u_{\mu} u_{v}\left\langle\left\langle A^{\mu}(x) A^{v}(y)\right\rangle\right\rangle$.
Here $u^{\mu}=\left(1,0_{\perp}, 1\right)$ is the light-like four vector along the $z$ axis. The gluon correlator $G$ may be expressed via the HTL gluon polarization operator. Making use of an elegant sum rule for the transverse and longitudinal HTL gluon selfenergies derived in [41] the function $P(\rho)$ may be written as [31]

$P(\boldsymbol{\rho})=\frac{g^{2} C_{F} T}{(2 \pi)^{2}} \int \mathrm{d} \mathbf{q}_{\perp}\left[1-\exp \left(i \rho \mathbf{q}_{\perp}\right)\right] C\left(\mathbf{q}_{\perp}\right)$,

$C\left(\mathbf{q}_{\perp}\right)=\frac{m_{D}^{2}}{\mathbf{q}_{\perp}^{2}\left(\mathbf{q}_{\perp}^{2}+m_{D}^{2}\right)}$,

where $m_{D}=g T\left[\left(N_{c}+N_{F} / 2\right) / 3\right]^{1 / 2}$ is the Debye mass. In [31] it was demonstrated that for the case without external field calculation of the spectrum given by (17) within the LCPI formalism with the use of (27), (28) is equivalent to solving the integral equation obtained in the AMY analysis [21] in the momentum representation. And the formulas (10), (11), (16) reproduce exactly the AMY photon emission rate.

In the approximation of static color Debye-screened scattering centers (in the sense of quark multiple scattering in the QGP) [42] the function $P(\rho)$ reads

$P(\rho)=\frac{n \sigma_{q \bar{q}}(\rho)}{2}$,

where $n$ is the number density of the color centers, and

$\sigma_{q \bar{q}}(\rho)=C_{T} C_{F} \alpha_{s}^{2} \int \mathrm{d} \mathbf{q}_{\perp} \frac{\left[1-\exp \left(i \mathbf{q}_{\perp} \boldsymbol{\rho}\right)\right]}{\left(\mathbf{q}_{\perp}^{2}+m_{D}^{2}\right)^{2}}$

is the well-known dipole cross section $[43,44]$ with $C_{T}$ being the color center Casimir operator.

Both for the HTL scheme (27), (28) and the static approximation (29), (30) at $\rho \lesssim 1 / m_{D}$ approximately $P(\rho) \propto \rho^{2}$. At $\rho \ll 1 / m_{D}$ the function $P(\rho)$ in the static model differs from that in the HTL scheme just by the normalization factor $\frac{\pi^{2}}{6 \cdot 1.202}\left(1+N_{f} / 6\right) /\left(1+N_{f} / 4\right) \approx 1.19\left(\right.$ for $\left.N_{f}=2.5\right)$. The replacement of the factor $1 /\left(q^{2}+m_{D}^{2}\right)^{2}$ in the dipole cross section in the static model by $1 /\left[q^{2}\left(q^{2}+m_{D}^{2}\right)\right]$ in the HTL scheme leads to unlimited growth of $P(\rho)$ at large $\rho$ (due to zero magnetic mass in the HTL approximation), while for static model $P(\rho)$ flattens at $\rho \gtrsim 1 / m_{D}$. However, this difference is not very important from the point of view of the photon emission, because the contribution of the region $\rho \gtrsim 1 / m_{D}$ is relatively small (in the sense of the path integral representation of the Green function $\mathcal{K}$ entering to (17)).

We will work in the oscillator approximation

$P(\rho)=C_{p} \rho^{2}$,

which is widely used in jet quenching analyses [45-50]. The $C_{p}$ can be expressed via the transport coefficient $\hat{q}$ [38-40], 
describing gluon transverse momentum broadening in the QGP, as $C_{p}=\hat{q} C_{F} / 4 C_{A}$. In numerical calculations we use $\hat{q} \propto T^{3}$ and set $\hat{q}=0.2 \mathrm{GeV}^{3}$ at $T=250 \mathrm{MeV}$. This value is supported by estimate of $\hat{q}$ within the static model via the magnitude of the dipole cross section at $\rho \sim 1 / m_{q}$, which allows one to describe well the data on jet quenching in $A A$ collisions within the LCPI scheme [2-5]. It also agrees with the qualitative $\mathrm{pQCD}$ calculations of Ref. [51] that give $\hat{q} \sim 2 \varepsilon^{3 / 4}$, with $\varepsilon$ the QGP energy density) (it gives $\hat{q} \approx$ $14 T^{3}$ ). Note that the estimate obtained in [51] agrees with the relation between $\hat{q}$ and the ratio of the shear viscosity to the entropy density $\eta / s$

$\hat{q} \sim 1.25 T^{3} s / \eta$

obtained in [52] if one takes the quantum limit value $\eta / s=$ $1 / 4 \pi[53]$.

\subsection{Photon spectrum in the oscillator approximation}

For the quadratic $P(\rho)$ the Hamiltonian (20) takes the oscillator form (we omit arguments of functions for brevity, where possible)

$\hat{\mathcal{H}}=-\frac{1}{2 M}\left(\frac{\partial}{\partial \boldsymbol{\rho}}\right)^{2}+\frac{M \Omega^{2} \boldsymbol{\rho}^{2}}{2}-\mathbf{f} \rho$

with

$\Omega=\sqrt{-i C_{p} x^{2} / M}$

The Green function for the Hamiltonian (33) is known explicitly (see, for example, [54])

$\mathcal{K}\left(\boldsymbol{\rho}_{2}, z_{2} \mid \boldsymbol{\rho}_{1}, z_{1}\right)=\frac{M \Omega}{2 \pi i \sin (\Omega z)} \exp \left[i S_{c l}\left(\boldsymbol{\rho}_{2}, z_{2} \mid \boldsymbol{\rho}_{1}, z_{1}\right)\right]$,

where $z=z_{2}-z_{1}$, and $S_{c l}$ is the classical action. The action can be written as a sum $S_{c l}=S_{o s c}+S_{f}$ with

$$
\begin{aligned}
& S_{o s c}\left(\boldsymbol{\rho}_{2}, z_{2} \mid \boldsymbol{\rho}_{1}, z_{1}\right) \\
& \quad=\frac{M \Omega}{2 \sin (\Omega z)}\left[\cos (\Omega z)\left(\boldsymbol{\rho}_{1}^{2}+\boldsymbol{\rho}_{2}^{2}\right)-2 \boldsymbol{\rho}_{1} \boldsymbol{\rho}_{2}\right], \\
& S_{f}\left(\boldsymbol{\rho}_{2}, z_{2} \mid \boldsymbol{\rho}_{1}, z_{1}\right)=\frac{M \Omega}{2 \sin (\Omega z)}\left[\mathbf{P}\left(\boldsymbol{\rho}_{1}+\boldsymbol{\rho}_{2}\right)-W\right],
\end{aligned}
$$

where

$$
\mathbf{P}=\frac{2 \mathbf{f}[1-\cos (\Omega z)]}{M \Omega^{2}},
$$

$W=\frac{2 \mathbf{f}^{2}}{M^{2} \Omega^{4}}\left[1-\cos (\Omega z)-\frac{\Omega z \sin (\Omega z)}{2}\right]$.

Then, after including the vacuum term in (17), a simple calculation gives

$\frac{\mathrm{d} P}{\mathrm{~d} x \mathrm{~d} L}=2 V(x)\left(I_{o s c}+\Delta I\right)$.

Here $I_{\text {osc }}$ corresponds to the pure oscillator case $(\mathbf{f}=0)$. It reads

$$
I_{\mathrm{osc}}=\frac{1}{\pi} \operatorname{Re} \int_{0}^{\infty} \mathrm{d} z\left[\frac{1}{z^{2}}-\left(\frac{\Omega}{\sin (\Omega z)}\right)^{2}\right] \exp \left(-i \frac{z}{\lambda_{f}}\right) .
$$

$\Delta I$ gives the synchrotron correction. It can be written as a sum $\Delta I=I_{1}+I_{2}$ with

$$
\begin{aligned}
I_{1}= & \frac{1}{\pi} \operatorname{Re} \int_{0}^{\infty} \mathrm{d} z\left(\frac{\Omega}{\sin (\Omega z)}\right)^{2}[1-\exp (-U)] \\
& \times \exp \left(-i \frac{z}{\lambda_{f}}\right), \\
I_{2}= & \frac{1}{\pi} \operatorname{Re} \int_{0}^{\infty} \mathrm{d} z \frac{i M \Omega^{3}}{8 \sin ^{3}(\Omega z)} \mathbf{P}^{2} \exp \left(-U-i \frac{z}{\lambda_{f}}\right),
\end{aligned}
$$

where

$U=\frac{i M \Omega W}{2 \sin (\Omega z)}$.

For numerical calculations it is convenient to introduce the dimensionless integrals

$\bar{I}_{\mathrm{osc}, 1,2}=\frac{\pi}{|\Omega|} I_{\mathrm{osc}, 1,2}$,

and to use the dimensionless integration variable $\tau=$ $z|\Omega| \exp (i \pi / 4)$. Then we obtain for $\bar{I}_{\mathrm{osc}, 1,2}$

$$
\begin{aligned}
& \bar{I}_{\text {osc }}(\kappa)=\operatorname{Re} \\
& \quad \times \int_{0}^{\infty} \frac{\mathrm{d} \tau \exp (i \pi / 4)}{\tau^{2}}\left(1-\frac{\tau^{2}}{\sinh ^{2} \tau}\right) \exp \left(-\frac{(1+i) \tau}{\sqrt{2} \kappa}\right),
\end{aligned}
$$

$$
\begin{aligned}
& \bar{I}_{1}(\kappa, \phi)=\operatorname{Re} \\
& \quad \times \int_{0}^{\infty} \frac{\mathrm{d} \tau \exp (i \pi / 4)}{\sinh ^{2} \tau}[1-\exp (-U)] \exp \left(-\frac{(1+i) \tau}{\sqrt{2} \kappa}\right),
\end{aligned}
$$




$$
\begin{aligned}
\bar{I}_{2}(\kappa, \phi)= & \frac{\phi}{2} \operatorname{Re} \int_{0}^{\infty} \mathrm{d} \tau \frac{(1-\cosh \tau)^{2}}{\sinh ^{3} \tau} \\
& \times \exp \left(-\frac{(1+i) \tau}{\sqrt{2} \kappa}-U\right),
\end{aligned}
$$

where now

$U=\frac{(1-i) \phi}{2 \sqrt{2}}[\tau-2 \tanh (\tau / 2)]$

and the dimensionless parameters $\kappa$ and $\phi \operatorname{read} \kappa=\lambda_{f}|\Omega|$, $\phi=\mathbf{f}^{2} / M|\Omega|^{3}$.

In the low density limit $(\kappa \rightarrow 0) \bar{I}_{\mathrm{osc}}(\kappa) \approx \kappa / 3$. The higher order terms in $\kappa$ describe the LPM effect. The ratio of $\bar{I}_{\mathrm{Osc}}$ to the leading order term gives the LPM suppression factor

$S_{\mathrm{LPM}}=3 \bar{I}_{\mathrm{Osc}} / \kappa$.

From (46), (50) one can obtain for two limiting cases of strong $(\kappa \gg 1)$ and weak $(\kappa \ll 1)$ LPM effect [27-30]:

$S_{\mathrm{LPM}} \approx \frac{3}{\kappa \sqrt{2}}(\kappa \gg 1) \quad S_{\mathrm{LPM}} \approx 1-\frac{16 \kappa^{4}}{21}(\kappa \ll 1)$.

In the limit $\Omega \rightarrow 0 I_{\text {osc }}=0$ and the integrals $I_{1,2}$ (42), (43) take the form (we denote them $I_{1,2}^{s}$ )

$I_{1}^{s}=\frac{1}{\pi} \operatorname{Re} \int_{0}^{\infty} \frac{\mathrm{d} z}{z^{2}} \exp \left(-i \frac{z}{\lambda_{f}}\right)\left[1-\exp \left(-i \frac{\mathbf{f}^{2} z^{3}}{24 M}\right)\right]$,

$I_{2}^{s}=\frac{1}{\pi} \operatorname{Re} \int_{0}^{\infty} \mathrm{d} z \frac{i \mathbf{f}^{2} z}{8 M} \exp \left(-i \frac{z}{\lambda_{f}}-i \frac{\mathbf{f}^{2} z^{3}}{24 M}\right)$.

Similarly to the case of $I_{1,2}(42),(43)$ it is convenient to go from (52), (53) to dimensionless integrals. Now we define them as

$\bar{I}_{1,2}^{s}=\pi \lambda_{f} I_{1,2}^{s}$.

Using the dimensionless integration variable $\tau=z \exp$ $(i \pi / 4) / \lambda_{f}$ from (42), (43) taking the limit $\Omega \rightarrow 0$ we obtain

$$
\begin{aligned}
& \bar{I}_{1}^{s}\left(\phi_{s}\right)=\operatorname{Re} \\
& \quad \times \int_{0}^{\infty} \frac{i d \tau}{\tau}\left[\exp \left(-\frac{(1-i) \phi_{s} \tau^{3}}{\sqrt{2}}\right)-1\right] \exp \left(-\frac{(1+i) \tau}{\sqrt{2}}\right),
\end{aligned}
$$

$$
\bar{I}_{2}^{s}\left(\phi_{s}\right)=6 \phi_{s} \operatorname{Re} \int_{0}^{\infty} \mathrm{d} \tau \tau \exp \left(-\frac{(1+i) \tau}{\sqrt{2}}-\frac{(1-i) \phi_{s} \tau^{3}}{\sqrt{2}}\right),
$$

where $\phi_{s}=\mathbf{f}^{2} \lambda_{f}^{3} / 24 M$. The functions (55), (56) may be expressed via the Airy function, $\operatorname{Ai}(z)=\frac{1}{\pi} \sqrt{\frac{z}{3}} K_{1 / 3}\left(2 z^{3 / 2} / 3\right)$ (here $K_{1 / 3}$ is the Bessel function),

$\bar{I}_{1}^{s}\left(\phi_{s}\right)=-\pi \int_{z}^{\infty} \mathrm{d} t \operatorname{Ai}(t)$

$\bar{I}_{2}^{s}\left(\phi_{s}\right)=-\frac{2 \pi}{z} \mathrm{Ai}^{\prime}(z)$,

where $z=1 /\left(3 \phi_{S}\right)^{1 / 3}$. Our probability of photon emission in the limit $\Omega \rightarrow 0$ is reduced to the well-known quasiclassical formula for the synchrotron spectrum $[55,56]$ in QED.

For $\gamma \rightarrow q \bar{q}$ one can obtain similar formulas. But now $M(x)=E_{\gamma} x(1-x)(x$ is the quark fractional momentum) $\epsilon^{2}=m_{q}^{2}-m_{\gamma}^{2} x(1-x), \mathbf{f}=z_{q} \mathbf{F}$, and

$V(x)=z_{q}^{2} \alpha_{e m} N_{c}\left[x^{2}+(1-x)^{2}\right] / 2$,

$\Omega=\sqrt{-i C_{p} / M}$.

The factor $N_{c}$ in (59) accounts for summing over the quark color indices for $\gamma \rightarrow q \bar{q}$ process. For $q \rightarrow \gamma q$ it does not appear in (19) since the sum over the quark color states is included in the factor $d_{b r}$ in (11).

Note that for the contribution of multiple scattering alone the oscillator approximation is equivalent to Migdal's calculations in QED within the Fokker-Planck approximation [36]. The oscillator approximation can lead to large errors in description of the gluon/photon emission from fast partons produced in hard reactions in the regime when the formation length is much bigger than the QGP size [9,57]. In this regime the oscillator approximation underestimates strongly the gluon/photon spectrum. However, this problem does not arise for the photon emission by the thermal quarks. In this case we have a situation similar to that for the photon emission from a quark propagating in an infinite medium. In this regime the errors of the oscillator approximation should not be large.

\section{Photon spectrum in $A A$ collisions}

\subsection{Integration over space-time coordinates}

For the $A A$ collision at a given impact parameter $b$ the thermal contribution to the photon spectrum $d N / d y d \mathbf{k}_{T}$ (we will consider the central rapidity region $y=0$ ) can be written as 
$\frac{\mathrm{d} N}{\mathrm{~d} y \mathrm{~d} \mathbf{k}_{T}}=\int \mathrm{d} t \mathrm{~d} V \omega^{\prime} \frac{\mathrm{d} N\left(T^{\prime}, F^{\prime}, k^{\prime}\right)}{\mathrm{d} t^{\prime} \mathrm{d} V^{\prime} \mathrm{d} \mathbf{k}^{\prime}}$,

where primed quantities correspond to the comoving frame, and $\omega^{\prime}=k^{\prime}=\left|\mathbf{k}^{\prime}\right|$ (here we consider a photon as a massless particle). In (61) we write explicitly the arguments of the photon emission rate in the comoving frame. The argument $F^{\prime}$ is the absolute value of the transverse (to the direction of the emitted photon) Lorentz force acting on a particle with electric charge $e$. Note that the photon emission rate in the comoving frame does not depend directly on the azimuthal direction of the photon momentum, and the angular dependence of the left-hand side of (61) stems solely from the dependence of the photon emission rate $d N\left(T^{\prime}, F^{\prime}, k^{\prime}\right) / \mathrm{d} t^{\prime} \mathrm{d} V^{\prime} \mathrm{d} \mathbf{k}^{\prime}$ on the righthand side on the photon momentum $k^{\prime}$ and on the Lorentz force $F^{\prime}$. The value of $\omega^{\prime}$ may be written via the photon four momentum $k^{\mu}=\left(\omega, \mathbf{k}_{T}, 0\right)$ in the c.m. frame of the $A A$ collision as

$\omega^{\prime}=u^{\mu} k_{\mu}$,

where

$u^{\mu}=\left(\frac{1}{\sqrt{1-\mathbf{v}^{2}}}, \frac{\mathbf{v}}{\sqrt{1-\mathbf{v}^{2}}}\right)$

is the four velocity of the QGP cell. The value of $F^{\prime}$ also can be expressed via the photon four momentum $k^{\mu}$ and the four velocity of the QGP cell. In the matter comoving frame

$F^{\prime}=e\left|\mathbf{E}_{\perp}^{\prime}+\left[\mathbf{n}^{\prime} \times \mathbf{B}^{\prime}\right]\right|$,

where $\mathbf{n}^{\prime}$ is the unit vector in the direction of the photon momentum, $\mathbf{E}_{\perp}$ is transverse (to the vector $\mathbf{n}^{\prime}$ ) component of the electric field. In terms of the electromagnetic field tensor $F^{\mu v}$ in the c.m. frame of the $A A$ collisions (64) can be written as

$F^{\prime}=e \sqrt{-L^{\mu} L_{\mu}}$,

where

$L^{\mu}=\frac{F^{\mu v} k_{\mu}}{u^{\delta} k_{\delta}}$.

As usual we write the four volume integration in (61) changing the integration variables $t, z$ to the proper time $\tau$ and rapidity $Y$

$\tau=\sqrt{t^{2}-z^{2}}, \quad Y=\frac{1}{2} \ln \left(\frac{t+z}{t-z}\right)$.

In these coordinates

$\frac{\mathrm{d} N}{\mathrm{~d} y \mathrm{~d} \mathbf{k}_{T}}=\int \tau \mathrm{d} \tau \mathrm{d} Y \mathrm{~d} \rho \omega^{\prime} \frac{\mathrm{d} N\left(T^{\prime}, F^{\prime}, k^{\prime}\right)}{\mathrm{d} t^{\prime} \mathrm{d} V^{\prime} \mathrm{d} \mathbf{k}^{\prime}}$.
The use of the formulas (62), (65), (66) allows one to avoid the Lorentz transformations from the quantities in the c.m. frame of $A A$ collisions to the ones in the comoving frame of the QGP. It makes the calculations for an expanding QGP as simple as for a QGP at rest.

Note that from (62) it is clear that the $Y$-integration in (68) is dominated by the region $|Y-y| \lesssim 1$. This is because the photon emission rate in the QGP rest frame in the integrand in (68) falls rapidly with $k^{\prime}$, and from (62) one obtains $k^{\prime}=k \cdot \cosh (Y-y)$ (we neglect the transverse expansion). Since the dominating contribution in the $\tau$-integration in (68) comes from $\tau \lesssim 2-3 \mathrm{fm}$, the effective two volume for the integration over $t$ and $z$ is $\sim 5-10 \mathrm{fm}^{2}$. It is by a factor of $\sim 10-20$ smaller than that of [25], where the $t$ - and $z$ integrations have been performed for $T=$ const, and $\mathbf{v}=0$ (which gives $k=k^{\prime}$ ) over the region $|z|<t<10 \mathrm{fm}$.

\subsection{Model of the fireball}

It is widely believed that the plasma fireball is produced in $A A$ collisions after thermalization of the glasma color tubes created in interaction of the Lorentz-contracted nuclei [58]. The typical time of evolution of the glasma color fields is about several units of $1 / Q_{s}$, where $Q_{s}(\sim 1-1.5 \mathrm{GeV}$ for RHIC and LHC conditions [59]) is the saturation scale of the nuclear parton distributions. It means that even for a very fast thermalization of the glasma color fields one can apply the formulas obtained for the equilibrium QGP only at $\tau \gtrsim 0.2-$ $0.5 \mathrm{fm}$. The thermalization time $\tau \sim 0.2 \mathrm{fm}$ means practically instantaneous process of the glasma thermalization at $\tau \sim 1 / Q_{s}$, and does not seem to be realistic. Nevertheless, in some analyses of the photon production $[60,61]$ the authors use $\tau_{0}=0.2$ and $0.1 \mathrm{fm}$ for RHIC and LHC energies, respectively. But such small values do not have a theoretical justification. In the present analysis we use a more realistic value of $\tau_{0}=0.4 \mathrm{fm}$ used in the analysis [19]. To account for qualitatively the fact that the process of the QGP production is not instantaneous we take the entropy density $\propto \tau$ in the interval $0<\tau<\tau_{0}$. However, the contribution of this region is relatively small (due to the factor $\tau$ in the integrand in (68)).

We describe the plasma fireball in the thermalized stage at $\tau>\tau_{0}$ in the Bjorken model [62] without the transverse expansion that gives the entropy density $s \propto 1 / \tau$. For the ideal gas model with $s \propto T^{3}$ it gives $T=T_{0}\left(\tau_{0} / \tau\right)^{1 / 3}$ in the plasma phase. However, the lattice calculations show [37] that for the temperature range of interest $T \lesssim 500 \mathrm{MeV}$ the entropy density exhibits a significant deviation from the $s \propto T^{3}$ dependence. For this reason it seems reasonable [63] to determine the plasma temperature from the temperature dependence of the entropy density predicted by lattice calculations. In our analysis we determined $T$ from the entropy density obtained in [37]. At $T \sim(1-2) T_{c}$ it gives the temper- 
ature greater than that for the ideal gas dependence $s \propto T^{3}$ by $10-20 \%$. This relatively small increase in $T$ may be important for the photon emission rate, because its $k$-dependence comes mostly from the exponential factor $\exp (-k / T)$ (stemming from the Fermi distribution (12)), which at $k \gg T$ is sensitive even to a small variation of $T$.

In Bjorken's model the entropy density of the QGP at a given impact parameter vector $\mathbf{b}$ of the $A A$ collision can be written as

$s(\tau, \boldsymbol{\rho}, Y, \mathbf{b})=\frac{1}{\tau} \frac{\mathrm{d} S(\boldsymbol{\rho}, Y, \mathbf{b})}{\mathrm{d} \rho \mathrm{d} Y}$,

where $\mathrm{d} S / \mathrm{d} \rho \mathrm{d} Y$ is the distribution of the entropy in the impact parameter plane and rapidity. For simplicity we take a Gaussian distribution of the entropy in the rapidity

$\frac{\mathrm{d} S(\boldsymbol{\rho}, Y, \mathbf{b})}{\mathrm{d} \rho \mathrm{d} Y}=\frac{\mathrm{d} S(\boldsymbol{\rho}, Y=0, \mathbf{b})}{\mathrm{d} \rho \mathrm{d} Y} \exp \left(-Y^{2} / 2 \sigma_{Y}^{2}\right)$.

For Au $+\mathrm{Au}$ collisions at $\sqrt{s}=0.2 \mathrm{TeV}$ we take for the width in $Y \sigma_{Y}=2.63$, which allows one to reproduce qualitatively the experimental pseudorapidity distribution of the charged particles $\mathrm{d} N_{c h} / \mathrm{d} \eta$. However, the results are not sensitive to the exact choice of $\sigma_{y}$, because the dominating contribution to the $Y$-integral in (68) comes from $|Y| \lesssim 1$.

We calculate the initial density profile in the impact parameter plane of the entropy at the proper time $\tau_{0}$ assuming that it is proportional to the charged particle pseudorapidity density at $\eta=0$ calculated in the two component wounded nucleon Glauber model [64],

$\frac{\mathrm{d} N_{c h}(\boldsymbol{\rho}, \mathbf{b})}{\mathrm{d} \eta d \boldsymbol{\rho}}=\frac{\mathrm{d} N_{c h}^{p p}}{\mathrm{~d} \eta}\left[\frac{(1-\alpha)}{2} \frac{\mathrm{d} N_{p a r t}(\boldsymbol{\rho}, \mathbf{b})}{\mathrm{d} \boldsymbol{\rho}}+\alpha \frac{\mathrm{d} N_{c o l l}(\boldsymbol{\rho}, \mathbf{b})}{\mathrm{d} \boldsymbol{\rho}}\right]$

where $\mathrm{d} N_{c h}^{p p} / \mathrm{d} \eta$ is the pseudorapidity multiplicity density for $p p$ collisions, and

$$
\begin{aligned}
& \frac{\mathrm{d} N_{\text {part }}(\boldsymbol{\rho}, \mathbf{b})}{\mathrm{d} \boldsymbol{\rho}} \\
& =T_{A}(|\boldsymbol{\rho}-\mathbf{b} / 2|)\left[1-\exp \left(-\sigma_{p p} T_{A}(|\boldsymbol{\rho}+\mathbf{b} / 2|)\right)\right] \\
& \quad+T_{A}(|\boldsymbol{\rho}+\mathbf{b} / 2|)\left[1-\exp \left(-\sigma_{p p} T_{A}(|\boldsymbol{\rho}-\mathbf{b} / 2|)\right)\right],
\end{aligned}
$$

$\frac{\mathrm{d} N_{\text {coll }}(\boldsymbol{\rho}, \mathbf{b})}{\mathrm{d} \boldsymbol{\rho}}=\sigma_{p p} T_{A}(|\boldsymbol{\rho}-\mathbf{b} / 2|) T_{A}(|\boldsymbol{\rho}+\mathbf{b} / 2|)$.

Here $T_{A}(b)=\int \mathrm{d} z n_{A}\left(\sqrt{b^{2}+z^{2}}\right)$ is the nuclear profile function calculated with the Woods-Saxon nuclear distribution,

$n_{A}(r)=\frac{N}{1+\exp \left[\left(r-R_{A}\right) / a\right]}$, where $N$ is the normalization constant, $R_{A}=\left(1.12 A^{1 / 3}-\right.$ $\left.0.86 / A^{1 / 3}\right) \mathrm{fm}, a=0.54 \mathrm{fm}[65]$. In numerical calculations for $\mathrm{Au}+\mathrm{Au}$ collisions at $\sqrt{s}=0.2 \mathrm{TeV}$ we take $\mathrm{d} N_{c h}^{p p} / \mathrm{d} \eta=2.65$ and $\sigma_{p p}=35 \mathrm{mb}$ obtained by the UA1 collaboration [66] for non-single diffractive inelastic events. We take $\alpha=0.135$ [67], which allows one to describe well the data from STAR [68] on the centrality dependence of $\mathrm{d} N_{c h} / \mathrm{d} \eta$ in $\mathrm{Au}+\mathrm{Au}$ collisions at $\sqrt{s}=0.2 \mathrm{TeV}$. To fix the normalization of the entropy density we use the relation $\mathrm{d} S / \mathrm{d} Y / \mathrm{d} N_{c h} / \mathrm{d} \eta \approx 7.67$ obtained in [69]. For central $\mathrm{Au}+\mathrm{Au}$ collisions at $\sqrt{s}=0.2 \mathrm{TeV}$ this procedure gives the plasma temperature at the center of the fireball $T \approx 465 \mathrm{MeV}$ at $\tau=0.4 \mathrm{fm}$. In the space-time integral (68) we drop the points which formally give $T<T_{c}$ (we take $T_{c}=165 \mathrm{MeV}$ ) at $\tau=\tau_{0}$. We treat the crossover region at $T \sim T_{c}$ as a mixed phase, assuming that the entropy density in this phase $\propto 1 / \tau$ [62], and we account for only the QGP phase. However, the contribution of the space-time region with $T \sim T_{C}$ to the photon spectrum in $A A$ collisions is relatively small even at $k_{T} \sim 0.5 \mathrm{GeV}$. And at $k_{T} \gtrsim 1.5-2 \mathrm{GeV}$ the contribution of this space-time region is practically unimportant.

\subsection{Electromagnetic field in the fireball}

For computation of the synchrotron contribution to the photon emission rate we need to know the magnitude of the electromagnetic field in $A A$ collisions in the space-time region occupied by the QGP, i.e. even for very optimistic scenarios with a fast thermalization of the glasma color fields it means the $\tau$-region $\tau \gtrsim 0.2 \mathrm{fm}$. Presently, there is no consensus within the heavy ion community on the magnitude of the electromagnetic fields in the QGP at such times.

The magnetic field generated by the Coulomb fields of the colliding nuclei at $\mathbf{r}=0$ (the center of the fireball) has the only nonzero component $B_{y}$ (for a coordinate frame as shown in Fig. 1). At $t=0$ and $\mathbf{r}=0$ the magnetic field reads [23]

$e B_{y}(t=0, \mathbf{r}=0) \approx \gamma Z \alpha b / R_{A}^{3}$,

and at $t^{2} \gtrsim\left(R_{A}^{2}-b^{2} / 4\right) / \gamma^{2}\left(b\right.$ is assumed to be $\left.<2 R_{A}\right)$ it is approximately

$e B_{y}(t, \mathbf{r}=0) \approx \frac{\gamma Z \alpha b}{\left(b^{2} / 4+\gamma^{2} t^{2}\right)^{3 / 2}}$

For $t \gg R_{A} / \gamma$ in the region $\rho \ll t \gamma$ the field has a simple $\rho$-independent form,

$e B_{y}(t, \rho, z=0) \approx \frac{Z \alpha b}{\gamma^{2} t^{3}}$. 
The quantity $R_{A} / \gamma$ is very small: $\sim 0.06 \mathrm{fm}$ for $\mathrm{Au}+\mathrm{Au}$ collisions at RHIC energy $\sqrt{s}=0.2 \mathrm{TeV}$, and $\sim 0.004 \mathrm{fm}$ for $\mathrm{Pb}+\mathrm{Pb}$ collisions at LHC energy $\sqrt{s}=2.76 \mathrm{TeV}$. For $\mathrm{Au}+\mathrm{Au}$ collisions at $\sqrt{s}=0.2 \mathrm{TeV}$ (77) gives

$e B_{y}(t, \rho, z=0) \approx m_{\pi}^{2} \cdot 10^{-4} \frac{(b / 1 \mathrm{fm})}{(t / 1 \mathrm{fm})^{3}}$.

And for $\mathrm{Pb}+\mathrm{Pb}$ collisions at $\sqrt{s}=2.76 \mathrm{TeV}$ from (77) we obtain

$e B_{y}(t, \rho, z=0) \approx m_{\pi}^{2} \cdot 5.5 \cdot 10^{-7} \frac{(b / 1 \mathrm{fm})}{(t / 1 \mathrm{fm})^{3}}$.

From these relations we obtain at $t=0.2 \mathrm{fm} e B_{y} \approx$ $0.075 m_{\pi}^{2}$ and $4 \cdot 10^{-4} m_{\pi}^{2}$ for RHIC and LHC, respectively. Thus, even for very optimistic assumption on the QGP formation time, the magnitude of the magnetic field in the initial stage of the QGP phase turns out to be much smaller than that in the first instant of the $A A$ collision $(75)\left(\sim 3 m_{\pi}^{2}\left(b / R_{A}\right)\right.$ and $\sim 40 m_{\pi}^{2}\left(b / R_{A}\right)$ for RHIC and LHC, respectively). From the above one sees that from the point of view of the synchrotron contribution to the photon emission rate a potentially interesting case is $\mathrm{Au}+\mathrm{Au}$ collisions at $\mathrm{RHIC}$. For $\mathrm{Pb}+\mathrm{Pb}$ collisions at LHC the magnitude of the magnetic field in the plasma stage is clearly too small to generate significant synchrotron radiation.

The presence of the QGP may modify the electromagnetic fields at later times due to the conductivity of the QGP. There was an idea that the induced currents generated in the conducting QGP can significantly delay the decay of the magnetic field [70]. It is possible if the magnetic lines, at least partly, are frozen in the QGP similarly to the ordinary conducting materials [71]. However, the analysis performed in [72] for the QGP with zero velocity has shown that for realistic plasma conductivity the effect of the induced currents is not strong enough to delay considerably the decay of the magnetic field. The computations for a realistic expanding plasma fireball have been performed in [23]. There, by solving Maxwell's equations in the Milne coordinates $x^{\mu}=(\tau, \boldsymbol{\rho}, Y)$, it was shown that, formally, at $\tau \gtrsim 0.5-1$ $\mathrm{fm}$ the induced currents can generate significant electromagnetic fields at the center of the fireball that are much bigger than the electromagnetic fields originating from the protons of the colliding nuclei. However, for realistic values of the plasma conductivity, the electromagnetic fields generated by the induced currents in the fireball turn out to be in a deep quantum regime when the typical occupation numbers are small. In this regime the induced currents lead only to a rare emission of single photons (with a typical energy about several units of the inverse size of the fireball (i.e. $\sim 1 / R_{A}$ ). It is clear that such single-photon processes cannot lead to the thermal synchrotron radiation from the QGP. In this physical picture of the electromagnetic response of the QGP we are left only with the synchrotron radiation related to the electromagnetic field generated by the protons of the colliding nuclei. As was shown above at $\tau=0.2 \mathrm{fm}$ we have $e B_{y} \sim 0.1 m_{\pi}^{2}$ for $\mathrm{Au}+\mathrm{Au}$ collisions at $\sqrt{s} \sim 0.2 \mathrm{TeV}$, To make our estimates of the synchrotron contribution as optimistic as possible we perform calculations for $e B_{y}=m_{\pi}^{2}$. Note that this value is somewhat larger than the magnitude of the magnetic field obtained in the recent analysis [73], and than that used in calculations of [25].

\section{Numerical results}

In this section we discuss our numerical results on photon emission from the QGP. The numerical results are obtained for the quark quasiparticle mass for $\alpha_{s}=0.2$. The basic ingredients used to calculate the photon emission rate from the QGP are the $x$-spectra of $q \rightarrow \gamma q$ and $\gamma \rightarrow q \bar{q}$ transitions. In Fig. 2 we present the prediction for these spectra for $u$ quark for $E_{q, \gamma}=2 \mathrm{GeV}$ at $T=250$ and 500 $\mathrm{MeV}$, and $e B=m_{\pi}^{2}$. We show separately the contributions from multiple scattering and the effect of the magnetic field. For comparison we also show the results for the purely synchrotron spectrum (i.e., for $\hat{q}=0$ ). From Fig. 2 one sees that for $q \rightarrow \gamma q$ multiple scattering reduces strongly the synchrotron contribution at moderate values of $x$. However, even without this suppression the pure synchrotron contribution is much smaller than the contribution to the spectrum related to multiple scattering of quarks in the QGP. For the $\gamma \rightarrow q \bar{q}$ the pattern of interplay of the effects from magnetic field and multiple scattering is more complicated. At
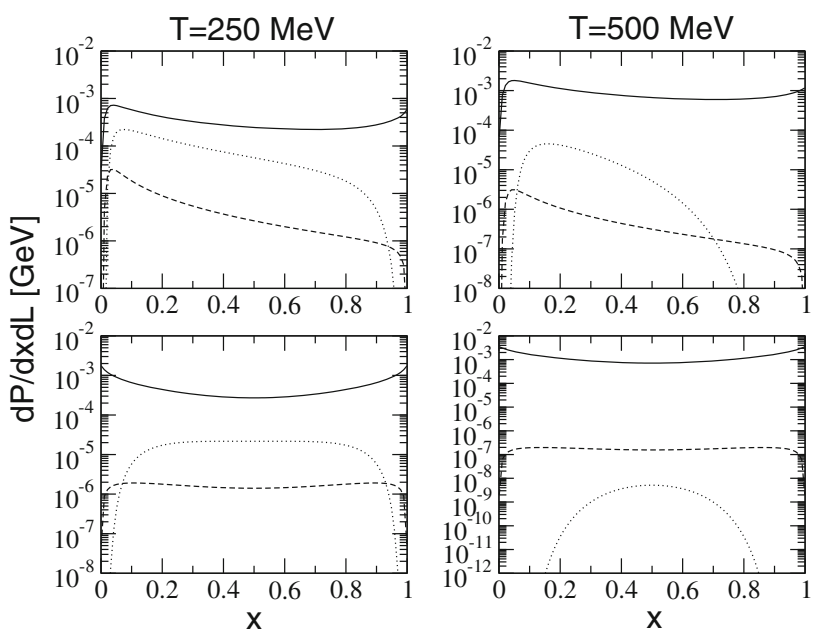

Fig. 2 The probability distribution $\mathrm{d} P / \mathrm{d} x \mathrm{~d} L$ for $q \rightarrow \gamma q$ (upper) at $E_{q}=2 \mathrm{GeV}$ and $\gamma \rightarrow q \bar{q}$ (lower) at $E_{\gamma}=2 \mathrm{GeV}$ at $T=250$ (left) and 500 (right) $\mathrm{MeV}$ for $u$ quark. Solid: the contribution of multiple scattering, dotted: the pure synchrotron contribution, dashed: the synchrotron contribution obtained with account for multiple scattering. The synchrotron contributions are computed for $e B=m_{\pi}^{2}$ 


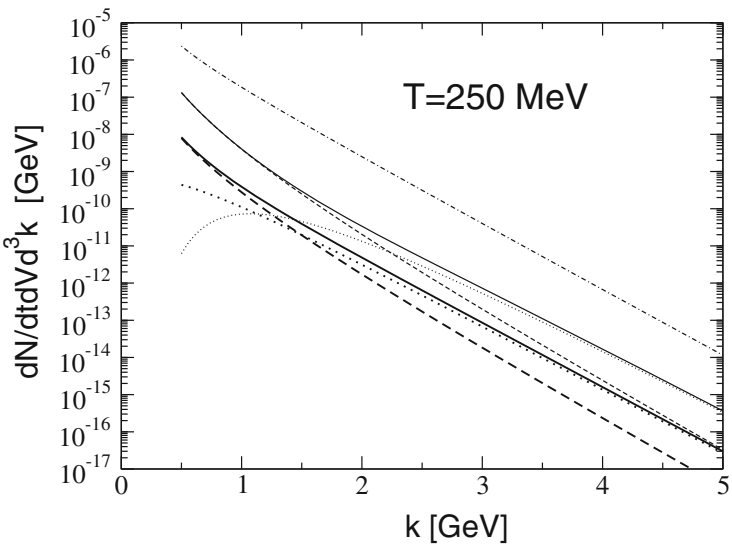

Fig. 3 The photon emission rate $\mathrm{d} N / \mathrm{d} t \mathrm{~d} V \mathrm{~d} \mathbf{k}$ in the QGP rest frame for $T=250$ (left) and 500 (right) MeV. Solid: the sum of the synchrotron contributions from $q \rightarrow \gamma q$ and $q \bar{q} \rightarrow \gamma$ processes, dashed: the synchrotron contribution from $q \rightarrow \gamma q$, dotted: the synchrotron contribution from $q \bar{q} \rightarrow \gamma$,dot-dashed: the sum of the contributions from $q \rightarrow \gamma q$ and $q \bar{q} \rightarrow \gamma$ transitions due to multiple scattering alone.

$T=250 \mathrm{MeV}$ at moderate $x$ the synchrotron contribution obtained accounting for multiple scattering is much smaller than the one obtained with $\hat{q}=0$. But at $T=500 \mathrm{MeV}$ multiple scattering enhances the synchrotron contribution. However, similarly to the $q \rightarrow \gamma q$ process, the synchrotron contribution turns out to be much smaller than the spectrum generated by quark multiple scattering alone.

In Fig. 3 we show the results of the computation of $d N / d t d V d \mathbf{k}$ for bremsstrahlung and annihilation and for their sum at $T=250$ and $500 \mathrm{MeV}$. As in Fig. 2 we present also the curves obtained neglecting the effect of multiple scattering $(\hat{q}=0)$. One can see that, similarly to Fig. 2, the contribution from multiple scattering alone is much bigger than the contribution of the synchrotron mechanism. The curves for the total synchrotron mechanism ( $q \rightarrow \gamma q$ plus $q \bar{q} \rightarrow \gamma)$ obtained accounting multiple scattering go considerably below the ones for the synchrotron contribution for $\hat{q}=0$. From Fig. 3 one sees that for the synchrotron mechanism with multiple scattering the contribution from $q \bar{q} \rightarrow \gamma$ process becomes larger than the one from $q \rightarrow \gamma q$ at $k \gtrsim 1.5 \mathrm{GeV}$ for $T=250 \mathrm{MeV}$ and at $k \gtrsim 3 \mathrm{GeV}$ for $T=500 \mathrm{MeV}$. Figure 3 shows that for a version with multiple scattering the contribution of the synchrotron mechanism turns out to be practically negligible as compared to the photon emission due to ordinary quark multiple scattering in the QGP.

In Figs. 4, 5, and 6 we present the results for the photon spectrum $\mathrm{d} N / \mathrm{d} y \mathrm{~d} \mathbf{k}_{T}=\left(1 / 2 \pi k_{T}\right) \mathrm{d} N / \mathrm{d} y \mathrm{~d} k_{T}$ (averaged over the azimuthal angle) stemming from both $q \rightarrow \gamma q$ and $q \bar{q} \gamma$ processes for $\mathrm{Au}+\mathrm{Au}$ collisions at $\sqrt{s}=0.2 \mathrm{TeV}$ for three centrality bins $0-20 \%, 20-40 \%$, and $40-60 \%$. The theoretical curves have been obtained integrating in (68) up to

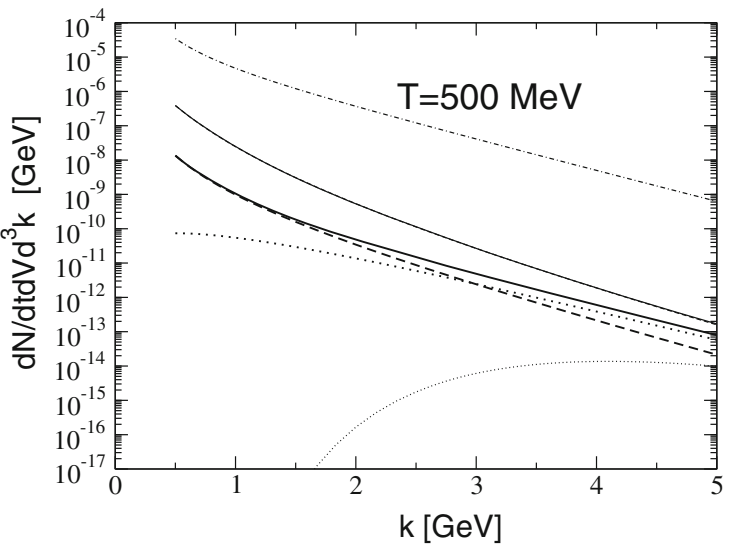

The thin solid, dashed, and dotted lines show the predictions for the pure synchrotron mechanism, and the thick ones show the predictions obtained with account for multiple scattering. The synchrotron contributions are computed for $e B=m_{\pi}^{2}$ (the magnetic field is assumed to be perpendicular to the photon momentum)

$\tau_{\max }=10 \mathrm{fm}$. The calculations with $\tau_{\max }=R_{A} \approx 6.4$ fm give very similar results at $k_{T} \gtrsim 1.5 \mathrm{GeV}$, and at $k_{T} \sim 0.5-0.75 \mathrm{GeV}$ the photon spectrum is reduced by $\sim 30-40 \%$. At $k_{T} \gtrsim 1.5 \mathrm{GeV}$ the results are only weakly sensitive to $\tau_{\max }$, because the main contribution at $k_{T} \gg T_{0}$ comes from the hottest space-time region of the QGP with $\tau$ up to several units of $\tau_{0}$. As in Fig. 3 we show the results for multiple scattering alone and for the two versions of the synchrotron contribution. One sees that multiple scattering strongly reduces the synchrotron contribution. It is important that for both the versions of the synchrotron contribution the effect is much smaller (by a factor of $\sim 10^{3}-10^{4}$ ) than the contribution from multiple scattering. Our calculations show that the azimuthal asymmetry $v_{2}$ for the synchrotron contribution alone is large $(\sim 0.5)$. However, since the relative contribution of the synchrotron mechanism to the photon emission rate is very small, its effect on the observable $v_{2}$ turns out to be negligible as well. We also present in Figs. 4, 5, 6 the sum of our contribution from multiple scattering and the LO contribution from $2 \rightarrow 2$ processes $q(\bar{q}) g \rightarrow \gamma q(\bar{q})$ and $q \bar{q} \rightarrow \gamma g$ in the form obtained in [21]. Although a detailed analysis of the experimental data on the direct photons in $A A$ collisions is not a purpose of this paper, in Figs. 4, 5, and 6 we also plot the data from PHENIX [16] obtained after subtraction of the $N_{\text {coll }}$ scaled photon spectrum for $p p$ collisions. One can see that the theoretical curves for the sum of the contribution from the collinear processes $q \rightarrow \gamma q$ and $q \bar{q} \gamma$ and the LO mechanisms underestimate the data by a factor of $\sim 2-4$. It is slightly bigger than found in the analysis [19] ( 1.5-3.5). However, in [19], in addition to the photon emission from the QGP, the radiation from the hadron 


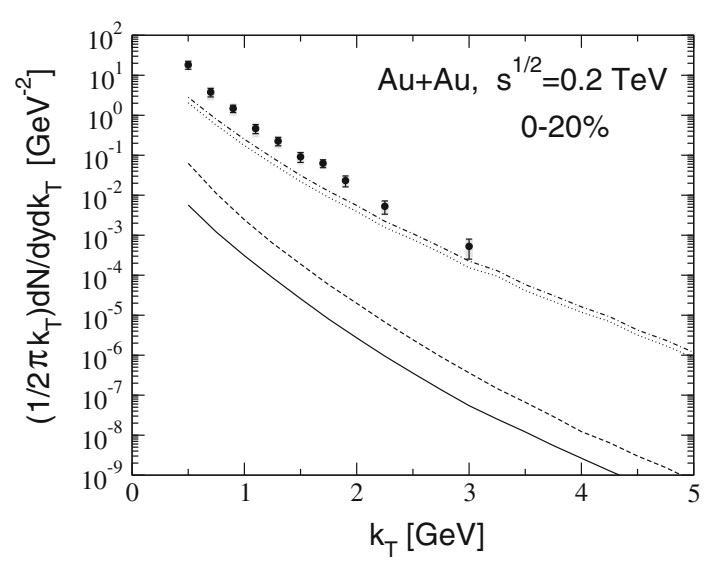

Fig. 4 The photon spectrum $\left(1 / 2 \pi k_{T}\right) d N / d y d k_{T}$ averaged over the azimuthal angle for $\mathrm{Au}+\mathrm{Au}$ collisions at $\sqrt{s}=0.2 \mathrm{TeV}$ in the 0 $20 \%$ centrality range. Solid the sum of the synchrotron contributions from $q \rightarrow \gamma q$ and $q \bar{q} \rightarrow \gamma$ processes calculated with account for multiple scattering, dashed the same as solid but without the effect of multiple scattering, dotted the contribution from $q \rightarrow \gamma q$ and $q \bar{q} \rightarrow \gamma$ processes due to quark multiple scattering alone, dot-dashed the sum of the contributions from $q \rightarrow \gamma q$ and $q \bar{q} \rightarrow \gamma$ processes due to quark multiple scattering and the contribution of the LO $2 \rightarrow 2$ processes in the form obtained in [21]. The data are from Ref. [16]

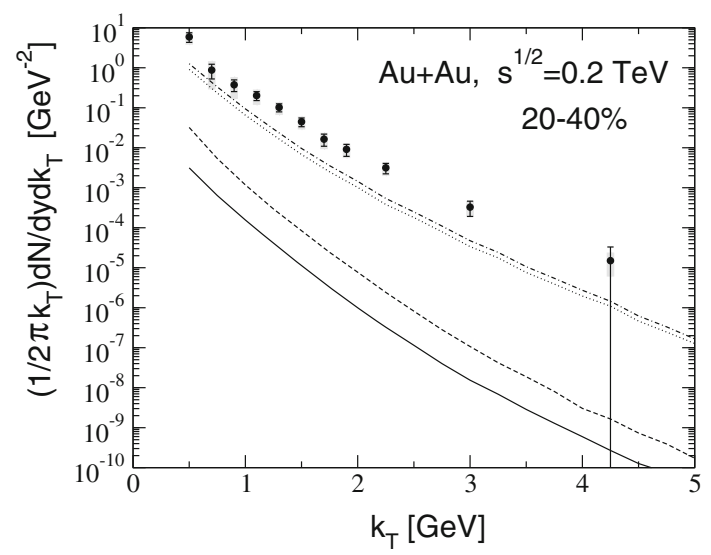

Fig. 5 Same as in Fig. 4 for $20-40 \%$ centrality bin

gas has been included, which is neglected in our calculations.

Thus, our calculations show that even for clearly too optimistic value of the magnetic field the effect of the synchrotron mechanism is very small. For more realistic field $e B \sim 0.1 m_{\pi}^{2}$ the synchrotron contribution is smaller by a factor of $\sim 10^{2}$. It leads to the conclusion that for RHIC and LHC conditions the synchrotron mechanism cannot be important neither for the azimuthally averaged photon spectrum nor for the azimuthal asymmetry $v_{2} .^{2}$

\footnotetext{
2 Assuming that our collinear formulas are qualitatively valid at $L_{f} / R_{L} \sim 1-3$ we have found that to obtain the photon $v_{2}$ with a magnitude comparable to the measured $v_{2}$ at $k_{T} \sim 1-3 \mathrm{GeV}$ one should assume that $e B \sim(30-70) m_{\pi}^{2}$. However, such strong fields in the QGP
}

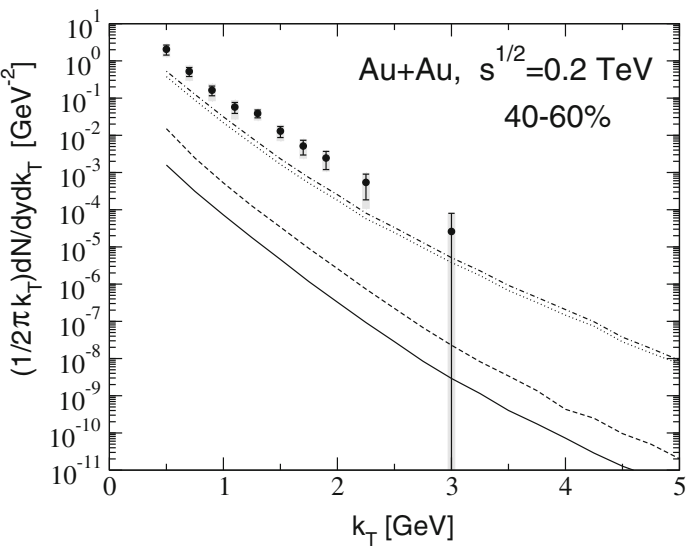

Fig. 6 Same as in Fig. 4 for $40-60 \%$ centrality bin

Our results are in strong disagreement with the recent analysis [25], where a rather large effect of the magnetic field was found. At $k_{T} \sim 1-3 \mathrm{GeV}$ our synchrotron contribution obtained without the effect of multiple scattering shown in Figs. $4,5,6$ by a factor of $\sim 10^{2}-10^{3}$ smaller than that from [25]. In [25] the $k_{T}$ photon spectrum was calculated for the QGP at rest and $T=$ const. As noted in the Introduction and in Sect. 3, this approximation should overestimate the photon emission rate (at least by a factor of $\sim 10$ ). However, the major source of the difference between our results and that of [25] is probably the different choice of the quark masses. We use for the quark mass the quark quasiparticle mass, while in [25] the current quark masses have been used. The theoretical basis for the use of the quasiparticle quark mass is the same as in the AMY scheme [21], where quarks acquire a dynamical thermal mass $\sim g T$ after the HTL resummation. As was demonstrated in Sect. 2 the adding of the external magnetic field does not change the physical picture of the collinear photon emission. We checked that for the photon momentum $k \sim 1-3 \mathrm{GeV}$ and $T \sim 250-500 \mathrm{MeV}$ the replacement of the thermal quark mass by the current one increases the pure synchrotron contribution by a factor of $\sim 10-200$. Note that for the synchrotron contribution obtained accounting for multiple scattering, i.e. for nonzero $\hat{q}$, the replacement of the thermal quark mass by the current one gives a relatively small enhancement $(\lesssim 1.5)$. It is connected with the fact that the coherence length of the photon/gluon emission in the presence of multiple scattering remains finite even for mass-

\footnotetext{
Footnote 2 Continued

stage are clearly unrealistic because they are by a factor of $\sim 10-20$ bigger than even the magnetic field in the first instant after the $A A$ collision (we consider $\mathrm{Au}+\mathrm{Au}$ collisions at $\sqrt{s}=0.2 \mathrm{TeV}$, and take $t \lesssim R_{A} / \gamma$ and $b \sim R_{A}$ ). Also, the electromagnetic energy density for such fields turns out to be too large (of the order of the thermal plasma energy at $T \sim 600 \mathrm{MeV}$ ) both for the RHIC and LHC conditions. These arguments show that the scenario with $e B \gg m_{\pi}^{2}$, which could formally give a reasonable agreement with experimental data on $v_{2}$, can be rejected.
} 
less partons. ${ }^{3}$ Note that just for this reason the parton energy loss is well defined in the massless limit [38-40]. It is worth noting that the fact that the synchrotron contribution in the presence of multiple scattering remains small even for massless quarks shows that it should be small also for the scenario of a strongly coupled QGP with a very small thermal quark mass [74].

\section{Summary}

We have developed a formalism for evaluation of the photon emission from the QGP with external electromagnetic field due to the collinear processes $q \rightarrow \gamma q$ and $q \bar{q} \rightarrow \gamma$. Within this formalism we have studied the effect of the magnetic field on the photon emission rate from the QGP in AA collisions for a realistic model of the plasma fireball. We showed that multiple scattering reduces considerably the effect of the magnetic field. We found that even for an extremely optimistic assumption on the magnitude of the magnetic field $\left(e B \sim m_{\pi}^{2}\right)$ the effect of magnetic field on the photon emission in $A A$ collisions is very small. For more realistic fields $\left(e B \sim 0.1 m_{\pi}^{2}\right)$ the effect is practically negligible. For this reason, we conclude that the synchrotron mechanism cannot lead to a considerable azimuthal asymmetry in the photon emission rate in $A A$ collisions. Our calculations show that due to multiple scattering the synchrotron contribution is small even for massless quarks. For this reason for the scenario of a strongly coupled QGP with a very small thermal quark mass [74] the effect of the magnetic field on the photon emission should remain small.

Acknowledgements I thank P. Aurenche for useful discussions in the initial stage of this work. I am grateful to K. Tuchin for informing me about the values of parameters used in the numerical calculations of [25]. This work has been supported by the RScF Grant 16-12-10151.

Open Access This article is distributed under the terms of the Creative Commons Attribution 4.0 International License (http://creativecomm ons.org/licenses/by/4.0/), which permits unrestricted use, distribution, and reproduction in any medium, provided you give appropriate credit to the original author(s) and the source, provide a link to the Creative Commons license, and indicate if changes were made. Funded by SCOAP ${ }^{3}$.

\section{References}

1. K.C. Zapp, F. Krauss, U.A. Wiedemann, JHEP 1303, 080 (2013). arXiv:1212.1599

2. B.G. Zakharov, JETP Lett. 93, 683 (2011). arXiv: 1105.2028

3. B.G. Zakharov, JETP Lett. 96, 616 (2013). arXiv:1210.4148

4. J. Phys, G 40, 085003 (2013). arXiv:1304.5742

5. J. Phys, G 41, 075008 (2014). arXiv:1311.1159

\footnotetext{
$\overline{3}$ In terms of Eq. (5) it means that the quantity $S_{\mathrm{LPM}} / m_{q}^{2}$ is finite in the limit $m_{q} \rightarrow 0$
}

6. J. Xu, A. Buzzatti, M. Gyulassy, JHEP 1408, 063 (2014). arXiv: 1402.2956

7. K.M. Burke et al., JET Collaboration. Phys. Rev. C 90, 014909 (2014). arXiv: 1312.5003

8. P.F. Kolb, U.W. Heinz, arXiv:nucl-th/0305084

9. B.G. Zakharov, JETP Lett. 73, 49 (2001). arXiv:hep-ph/0012360

10. S. Caron-Huot, C. Gale, Phys. Rev. C 82, 064902 (2010). arXiv: 1006.2379

11. U. Heinz, R. Snellings, Ann. Rev. Nucl. Part. Sci. 63, 123 (2013). arXiv: 1301.2826

12. H. Song, S.A. Bass, U. Heinz, T. Hirano, Phys. Rev. C 83, 054910 (2011), Erratum. Phys. Rev. C 86, 059903 (2012). arXiv: 1101.4638

13. E.V. Shuryak, Phys. Lett. B 78, 150 (1978)

14. A. Adare et al., PHENIX Collaboration, Phys. Rev. Lett. 104, 132301 (2010). arXiv:0804.4168

15. A. Adare et al., PHENIX Collaboration, Phys. Rev. Lett. 109, 122302 (2012). arXiv: 1105.4126

16. A. Adare et al., PHENIX Collaboration, Phys. Rev. C 91, 064904 (2015). arXiv: 1405.3940

17. J. Adam et al. [ALICE Collaboration], Phys. Lett. B754, 235 (2016) arXiv: 1509.07324

18. C. Shen, arXiv: 1601.02563

19. J.-F. Paquet et al., Phys. Rev. C 93, 044906 (2016). arXiv: 1509.06738

20. K. Dusling, Nucl. Phys. A 839, 70 (2010). arXiv:0903.1764

21. P.B. Arnold, G.D. Moore, L.G. Yaffe, JHEP 0112, 009 (2001). arXiv:hep-ph/0111107

22. G. Basar, D. Kharzeev, V. Skokov, Phys. Rev. Lett. 109, 202303 (2012). arXiv:1206.1334

23. B.G. Zakharov, Phys. Lett. B 737, 262 (2014). arXiv:1404.5047

24. V.V. Goloviznin, A.M. Snigirev, G.M. Zinovjev, JETP Lett. 98, 61 (2013). arXiv: 1209.2380

25. K. Tuchin, Phys. Rev. C 91, 014902 (2015). arXiv:1406.5097

26. R. Baier, H. Nakkagawa, A. Niegawa, K. Redlich, Z. Phys. C 53, 433 (1992)

27. B.G. Zakharov, JETP Lett. 63, 952 (1996)

28. B.G. Zakharov, JETP Lett. 65, 615 (1997)

29. B.G. Zakharov, JETP Lett. 70, 176 (1999)

30. B.G. Zakharov, Phys. Atom. Nucl. 61, 838 (1998)

31. P. Aurenche, B.G. Zakharov, JETP Lett. 85, 149 (2007). arXiv:hep-ph/0612343

32. P. Aurenche, F. Gelis, H. Zaraket, Phys. Rev. D 61, 116001 (2000). arXiv:hep-ph/9911367

33. B.G. Zakharov, JETP Lett. in press arXiv:1607.04314

34. B.G. Zakharov, JETP Lett. 88, 475 (2008). arXiv:0809.0599

35. L.D. Landau, I.Y. Pomeranchuk, Dokl. Akad. Nauk SSSR92, 535, $735(1953)$

36. A.B. Migdal, Phys. Rev. 103, 1811 (1956)

37. S. Borsanyi et al., JHEP 1011, 077 (2010). arXiv:1007.2580

38. R. Baier, Y.L. Dokshitzer, A.H. Mueller, S. Peigné, D. Schiff, Nucl. Phys. B 483, 291 (1997)

39. R. Baier, Y.L. Dokshitzer, A.H. Mueller, S. Peigné, D. Schiff, Nucl. B 484, 265 (1997)

40. R. Baier, Y.L. Dokshitzer, A.H. Mueller, D. Schiff, Nucl. Phys. B 531, 403 (1998)

41. P. Aurenche, F. Gelis, H. Zaraket, JHEP 0205, 043 (2002)

42. M. Gyulassy, X.N. Wang, Nucl. Phys. B 420, 583 (1994)

43. N.N. Nikolaev, B.G. Zakharov, Z. Phys. C 49, 607 (1991)

44. N.N. Nikolaev, B.G. Zakharov, Z. Phys. C 53, 331 (1992)

45. R. Baier, Y.L. Dokshitzer, A.H. Mueller, D. Schiff, JHEP 0109, 033 (2001). arXiv:hep-ph/0106347

46. R. Baier, D. Schiff, JHEP 0609, 059 (2006). arXiv:hep-ph/0605183

47. C.A. Salgado, U.A. Wiedemann, Phys. Rev. Lett. 93, 042301 (2004). arXiv:hep-ph/0310079

48. N. Armesto, A. Dainese, C.A. Salgado, U.A. Wiedemann, Phys. Rev. D 71, 054027 (2005). arXiv:hep-ph/0501225 
49. J.-P. Blaizot, F. Dominguez, E. Iancu, Y. Mehtar-Tani, JHEP 1301, 143 (2013). arXiv: 1209.4585

50. P. Arnold, S. Iqbal, JHEP 1504, 070 (2015). arXiv:1501.04964

51. R. Baier, Nucl. Phys. A 715, 209 (2003). arXiv:hep-ph/0209038

52. A. Majumder, B. Muller, X.-N. Wang, Phys. Rev. Lett. 99, 192301 (2007). arXiv:hep-ph/0703082

53. P. Kovtun, D.T. Son, A.O. Starinets, Phys. Rev. Lett. 94, 111601 (2005). arXiv:hep-th/0405231

54. R.P. Feynman, A.R. Hibbs, Quantum Mechanics and Path Integrals (McGRAW-HILL Book Company, New York, 1965)

55. V.N. Baier, V.M. Katkov, JETP 26, 854 (1968)

56. V.B. Berestetski, E.M. Lifshits, L.P. Pitaevski, Quantum Electrodynamics(Landau Course of Theoretical Physics Vol. 4), (Pergamon Press, Oxford, 1979)

57. B.G. Zakharov, JETP Lett. 80, 1 (2004). arXiv:hep-ph/0405101

58. T. Lappi, L.D. McLerran, Nucl. Phys. A 772, 200 (2006). arXiv:hep-ph/0602189

59. T. Lappi, Eur. Phys. J. C 71, 1699 (2011). arXiv:1104.3725

60. J.K. Nayak, B. Sinha, Phys. Lett. B 719, 110 (2013). arXiv: 1210.3993

61. S. Mitra, P. Mohanty, S. Ghosh, S. Sarkar, Jan-e Alam, arXiv: 1303.0675

62. J.D. Bjorken, Phys. Rev. D 27, 140 (1983)
63. S. Turbide, R. Rapp, C. Gale, Phys. Rev. C 69, 014903 (2004). arXiv:hep-ph/0308085

64. D. Kharzeev, M. Nardi, Phys. Lett. B507, 121 (2001). arXiv:nucl-th/0012025

65. M. Rybczynski, G. Stefanek, W. Broniowski, P. Bozek, Comput. Phys. Commun. 185, 1759 (2014). arXiv:1310.5475

66. C. Albajar et al., UA1 Collaboration, Nucl. Phys. B 335, 261 (1990)

67. B.G. Zakharov, JETP Lett. in press arXiv:1605.06012

68. B.I. Abelev et al., STAR Collaboration, Phys. Rev. C 79, 034909 (2009). arXiv:0808.2041

69. B. Müller, K. Rajagopal, Eur. Phys. J. C 43, 15 (2005). arXiv:hep-ph/0502174

70. K. Tuchin, Adv. High Energy Phys. 2013, 490495 (2013). arXiv: 1301.0099

71. L.D. Landau, E.M. Lifshitz, Electrodynamics of Continuous Media (Addison-Wesley, Reading, Mass, 1960)

72. L. McLerran, V. Skokov, Nucl. Phys. A 929, 184 (2014). arXiv:1305.0774

73. K. Tuchin, Phys. Rev. C 93, 014905 (2016). arXiv:1508.06925

74. H. Nakkagawa, H. Yokota, K. Yoshida, Phys. Rev. D 85, 031902 (2012). arXiv:1111.0117 